\title{
Authentication of Bi-Level QR Code with Dynamic Pattern Generation
}

\author{
Karthik.B, Sriram.M, Sidharth Raj.R.S.
}

\begin{abstract}
This paper illustrates about a new Quick Response $(Q R)$ code to enhance data security with importance given to its wide use in information storage and authentication. The Bi-Level $Q R$ code add new abilities to standard QR Code which comprise of strengthening contemplating degree, which doesn't upset a similar old QR code examining procedure and development in privateers of primer $Q R$ code. The outstanding $Q R$ code utilized as first level of our Bi - Level $Q R$ code remains completely functional. It may be read with the aid of all standard application, without any restrict. Bi - Level QR code ensures privacy of records stored inside the 2d degree through a physical separation, between stages and reader utility skills.
\end{abstract}

Keywords : Bi-level, $Q R$ code, public level, private level, $P \& S$ process

\section{INTRODUCTION}

Nowadays, there are chief traits within the adoption of 2D Codes along with:he mandate by International Air Transport Association (IATA) for air terminals worldwide to embrace 2D standardized identification for traveler tickets by 2010 .

2) The appropriation of QR Code for patient ID by two driving emergency clinics in Singapore and all medical clinics in Hong Kong.

3) The utilization of 2D scanner tags/small scale codes for different applications in different divisions.

4) The utilization of QR code with cell phones in Japan and Korea.[1]

With the advent of Bi- level QR code the Authentication and message sharing capacity has been enhanced when compared to standard QR codes. The Bi-Level QR code has a unique Print-and - Scan $(\mathrm{P} \& S)$ process which eliminates duplication of the code. The assault .By this technique the assault strategy utilized in the standard QR code is limited all things considered. Here the continuous usage of the Bi - Level QR code is clarified by methods for an android application to book tickets in metro trains.[2]

\section{RELATEDWORK}

This area is part into two sub-areas (II-An, II-B). Area II - An arrangements with the portrayal of the most usually utilized Quick Response (QR) code.Section II - B deals with the proposed work $\mathrm{Bi}$ - Level QR code.[3]

\section{A. Quick Response (QR) code}

\section{Revised Manuscript Received on August 22, 2019.}

Karthik.B,Assistant Professor, Department of Electronics And Communication Engineering,, Bharath Institute of Higher Education and Research, Chennai, India. karthikguru33@gmail.com

Sriram.M. Assistant Professor, Department of Electronics And

Communication Engineering,, Bharath Institute of Higher Education and

Research, Chennai, India. gokulkrish1453@gmail.com

Sidharth Raj.R.S., Assistant Professor, Department of Electronics And Communication Engineering,, Bharath Institute of Higher Education and Research, Chennai, India. sidarthraj93@gmail.com
Dissimilar to the more established, one-dimensional scanner tags that had been The QR code is acknowledged by a 2-dimensional computerized image sensor and after that has been thoroughly broken down by a tailored processor, designed to be regularly checked using a thin light output. The operating system discovers 3 distinctive rectangles on the corners of the QR code photo, Using a smaller square (or different squares) near the fourth alcove to optimize the picture for the size, position, and mindset of the evaluation. Not at all like the more experienced, one-dimensional standard tags that were designed to be regularly filtered by the use of thin light combustion, the QR code is differentiated by a 2-dimensional automated image sensor and thereafter. The operator discovers 3 outstanding spaces on the edges of the QR code picture, using a smaller square (or countless squares) close the fourth niche to optimize the size, direction and layout of the picture.[4]

Technique used inside the QR code changed into that after a buyer channels the code he/she is composed toward a web site page and After that, a malicious record of transactions to the client's convenient marginalised of the client's knowledge. The confirmation is made in this document through the inspecting of Bi-level QR code by means of the versatile scanner application to recover open subtleties and through the remarkable scanner to recover the private subtleties...[5]

Information is put away in a QR In expressions which can be eight bits in length and utilize the Reed-Solomon botches amendment calculation with four configurable bumbles revision degrees. The better the error adjustment arrange, the less capacity ability. QR Code has botches revision usefulness to fix realities if the code is messy or harmed. Four errors adjustment levels are accessible for clients to pick as indicated by the working condition. according to the operating environment. The following figure illustrates the fixed patterns and the formatting info in the QR code.[6]

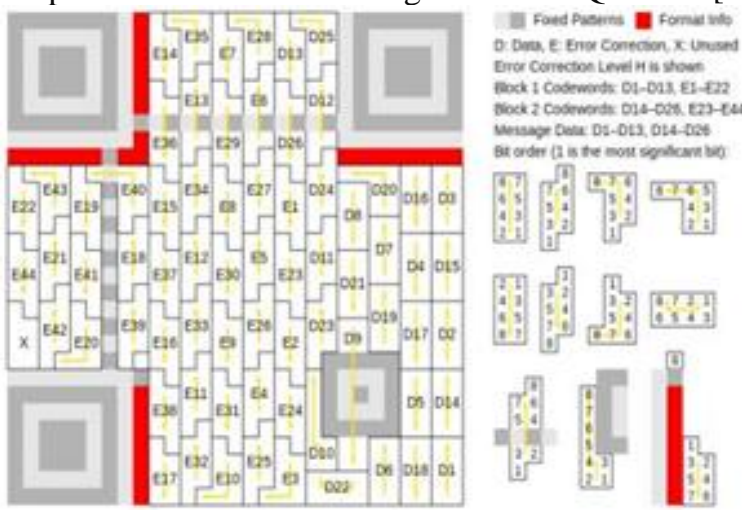

Fig 1. Fixed patterns and format info in QR code

Raising this stage improves botches redress ability yet in addition builds the measure of insights QR Code length. To pick 
mistakes adjustment degree, different components which incorporate the running complaint and QR Code size must be mulled over.[7]

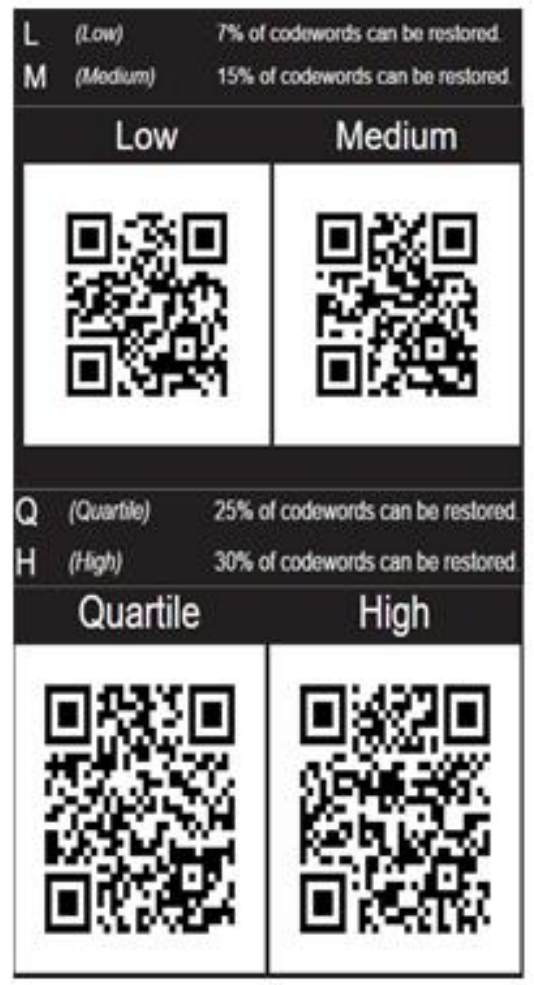

Fig 2. Efficiency of quick response code with respect to error correction

Level Q or $\mathrm{H}$ may be selected for the situation of the production plant in which the QR Code is dilapidated, while level L may be selected for a smooth situation with a gigantic metric of documents. Level M (15 per cent) is the most prevalent. extreme habitually chosen. The above photo records the rough mistakes amendment capacity at everything about 4 degrees:[11]

\section{B. Bi - Level QR code}

The Bi - Level Quick Response Code adds fresh capabilities to the normal QR code: 1. Supplementary phase analysis, which no longer interferes with the same ancient QR code technique of study. 2. Increase the garage ability of the preliminary QR code.[12]

The common QR code used as the first level of our Bi-Level QR code is still very helpful. It may be examined, without limitation, by any fashionable implementation.

Our Bi-Level QR code guarantees privacy of records stored inside the $2 \mathrm{~d}$ stage no longer handiest via applying a conventional numeric ciphering, but additionally with the aid of a bodily separation, among levels and reader application abilities. The Bi-Level QR code has two records stages:

1. The first level comprises of public data and can be studied by any QR code utility, i.e. I'm running OS, Android and scanner apps. That's why the suggested Bi-Level QR code satisfies all the latest QR code capacities.[13]

2. The second phase includes additional documents and is realized by replacing the black modules in the $\mathrm{QR}$ code with two accurate textured styles. The aggregate of these textured models makes it possible to encode and then reconstruct additional facts.[8]

\section{BI-LEVEL QUICK CODE RESPONSE WITH PUBLIC AND PRIVATE LEVELS}

Trans-level Barcode is the ordinary Txt file, both state and federal. Average Bar code generally includes only the public level, which shows all the data stored in it.. The primary aim of bi-level QR code is unnoticeable processing and distribution of personal data in QR code. This sub-section describes the steps of QR code formation, bi-level QR code storage capability and image processing. [9]

A Bi level QR code generationThis segment describes the structure of standard $\mathrm{QR}$ code with private level. After generating a standard QR code, private information is added to make it as a bi level QR code. Generation of standard QR code includes 7 steps, they are Data encoding, Data analysis, Data transmission coding, Configuration positioning, Final message structure, matrix, Data masking, Format and version data. [10]

The overview of Bi level QR code generation steps are illustrated in the fig. no. 3

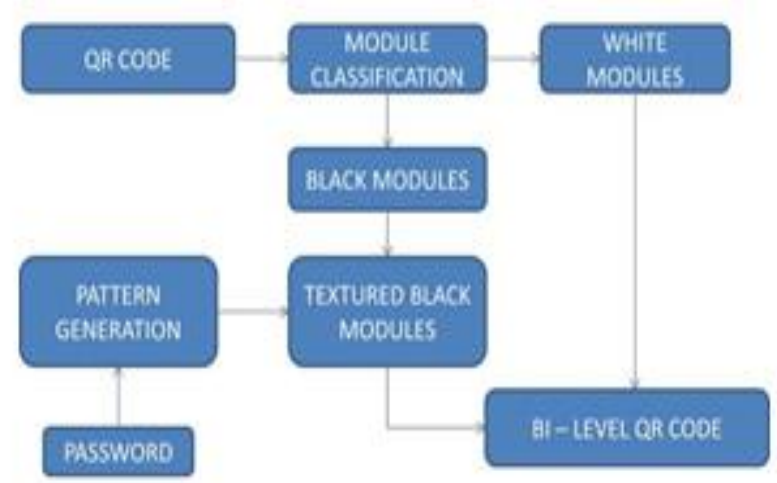

Fig 3. Bi-level QR code generation architecture diagram

In the first step, the The Url accesses a sequence of texts. The renowned QR system has four data information processing types that can be integer, alphanumeric, pixel and kanjiEach mode computes text as a sequence of pieces, and then each mode utilizes a separate approach to transform text to bits, and each processing method is configured to encode records with the quickest possible amount of bits... Therefore, first conduct a information assessment to evaluate not just whether your textual content can be encoded in numeric, alpha numeric, byte or kanji. Then choose the most effective mode for your code snippets.[14]

In statistics encoding stage, first step is to select errors correction stage. QR code makes use of Reed Solomon's mistake clarification code. This method produces a codeword error correction based on the encrypted data.

If the QR code viewer has not read the statistics properly, then blunders correction bytes are used. And additionally error correction code words can be used to accurate those mistakes. There are 4 tiers of blunders correction, they may be $\mathrm{L}, \mathrm{M}, \mathrm{Q}, \mathrm{H}$. The next step is to decide the model of the statistics. Then mode indicator is brought to it. Each mode of 
encryption has a four-bit mode marker that recognizes it.After that, encode the use of selected mode with the aid of breaking up into 8-bit codeword and pay bytes if important.

Next step is blunders correction coding This implies that after creating a statistic bit string,blunders correction codewords are generated the usage of those statistics bits. This method is called reed-solomon error correction. QR code scanner reads each of the data codewords and bug fixes the word code.. By evaluating those code words, QR code reader decide if it study the statistics successfully or not. If no longer, it can correct error the usage of those mistakes correction codewords. The information and error diagnostic code words produced in the previous step must be organized in the correct order. that's known as structure final message. For smaller codes, data and errors correction code words are used as it's miles and also interleaving is not necessary. In the case of large QR codes, documentation and encoding code words are produced in blocks and these blocks must be interlaced according to both the QR code configuration.

After statistics are arranged well inside the preceding step, module placement in matrix is performed on this step. Code words are arranged in a matrix in a chosen way.This step consists of feature styles. Function pattern consists of finder pattern, separator, alignment sample, timing pattern, darkish module. The finder pattern are 3 blocks within the corner of The QR code is at the top left, the top right and the lower part left. The dividers are the white space regions next to the dataset of the locator. The arrangement pattern is comparable to that of the locator sample, but lower and is placed through the code. The timing sample is a dotted line that links the sample of the finder. The Dark Component is a unmarried black segment that is continuously positioned next to the lowest left finder. Pattern.[16]

Certain patterns within The QR code matrix is hard to explore efficiently using QR code detectors. To overcome this, the which changes the QR code step by step with the exact pattern.. Then masked matrix is evaluated primarily based on four penalty rules with a view to decide which of those masks styles bring about the QR code with the fewest unwanted trends. The first reward rule provides the QR code with a punishment for each organisation of five or more equivalent colored components in a row or column.. The late penalty rule sets a penalty for each $2 * 2$ area of a certain colored components in the tesseract for the QR code. The third penalty rule provides the QR code with a big punishment if there are patterns. The look just like the finder styles.. Finally, the QR code should use the mask sequence that led to the smallest penalty rating.[17]

The very last step in well-known QR code is to feature layout and version data to the QR code via adding pixels specifically place of the code that had been left blank in previous step. The format pixel identifies the mistake correction degree and mask pattern getting used in this QR code. The version pixels encode the size of the QR code matrix and are best used in large QR codes. The format records string encodes which errors correction level and which masks sample is find inside the modern-day QR code. Since there are four viable errors correction tiers and 7 viable masks sample, consequently there are 28 viable layout records string. After generating layout string and model information, output might be the usual QR code.[18] QR code configuration describes 8 mask patterns, each of

After generating a Regular QR code, private information is added to the standard QR code to make it bi-level QR code.. The first step in bi level QR code is to separate black modules from the output of the standard QR code. And a pattern should be generated using the unique password which is given by the user. Then the generated pattern should be replaced in the particular place of the QR code in which place where the private information is encoded in it. After processing all these steps, the resultant will be the bi level QR code.[15]

\section{A. Storage capacity of Bi level}

The formula for determining the size of the $\mathrm{QR}$ code is $((\mathrm{v}-1) * 4)+21)$ everyplace $\mathrm{v}$ is a version of the $\mathrm{QR}$ program .If, for instance, the variant of the $\mathrm{QR}$ code is 10 , then the resultant of this formula for this example $(((10-1) * 4)+21)$ will be 57 modules by 57 modules. The data are stored in the finder The model of the Url and the place of the locator model can be extended as the top left part of the locator model seems to be at $(0,0)$, the upper left corner of the locator model is still at $([((((\mathrm{v}-1) * 4)+21)-7], 0)$ and the upper left corner of the locator model is still at $(0,[(\mathrm{v}-1) * 4)+21)-7])$. For a particular version, character capacity vary based on error correction level and text encoding modes. For example, in range 10, for mistake alteration level L, the capacity is 652 for numeric mode, 395 for alpha numeric mode, 271 for byte mode, 167 for kanji mode.

Since there are significant numbers of monochrome modules in the QR code, $\mathrm{N} 2 / 2$ is roughly the amount of black components in the Bar code. There are several three place labels and each tag has 33 components, there are only regarding (N2/2-3*33) black components in the Barcode that can be replaced by curved models.

Let $\mathrm{n}$ be the full set of digits in the catch all term, and $\mathrm{k}$ be the sum of the tip pieces.

\section{B. Recognition method}

The overview of the bi-level QR code reading method is shown in the fig. No. No. No. The bi-level QR code must first be fixed during the image analysis phase. Position tags are localized to assess the longitude of the position. Cartesian coordinates .Following this step, the Bi-level QR code has the right alignment and the initial size.

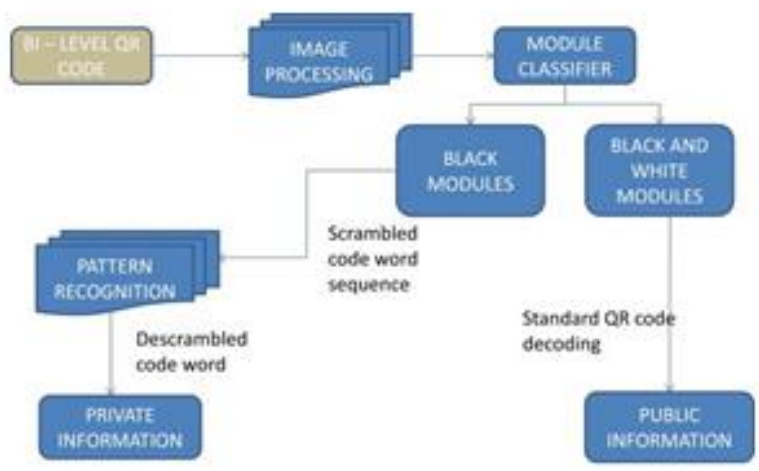

Fig 4. Bi-level qr code scanner architecture diagram

Published By:

Blue Eyes Intelligence Engineering 
The next step in reading process is module classification. Here, module classification is done by using threshold method. Specifically, global threshold is used This is determined as the average value of the whole $\mathrm{P} \& \mathrm{~S}$ Bi level $\mathrm{QR}$ code. If the median value of the $\mathrm{p}^{*} \mathrm{p}$ block pixels is lower than the limit value, this block is in the black category. Otherwise, the block is in the white group.

After separating the black and white modules, decoding of public information and recognition of private information are done in parallel. Decoding of public information is done by using standard QR code decoding algorithm. For pattern recognition, black modules are used. Black module class contain the textured patterns. The patterns which are replaced or scrambled during $\mathrm{QR}$ code generation are now descrambled to get the private information from the $\mathrm{QR}$ code.[19]

\section{EXPERIMENT RESULTS}

This section illustrates the improved authentication and how the dynamically generated pattern is included in the Bi-level QR code. However the storage capacity remains the same as proposed by the authors in[1]. The application scenario encompasses the ticket ID, the source, the destination, booking date and time, number of tickets and the amount as the public details. The private detail will include the payment ID which is encoded as the secret information.

The QR code version used here is the version 7 with the lower error correction level. This version has $45 \times 45$ modules and can encode bits of length 154 . The error correction is done by Golay correction code with the encoding $[11,6,5]$ with the digit length $n=11$, and where $k=6$ and the number of parity bits is $11-5=6$ [1].

\section{A.Bi-level QR code generation}

i. Generation of standard QR code: With the help of android studio IDE a standard QR code of version 7 is generated with the publicmessage as Mpub"Ash, code, source, destination, booking time, ticket count". The conversion or encoding of data in to QR code is done in the alphanumeric mode.

Step1: This mode involves splitting of the sequence into sets. For example, HELLO WORLD is split as HE, LL, O, WO, RL, D.

Step2:. Then add that amount to the second character representation amount. For example, the first pair in the HELLO WORLD is HE.H@17E@14.

After the steps in the previous paragraph, multiply the first number by 45 , and then add it to the second number: (45*17) $+14=779$ Now convert that number to an 11-bit binary string, with 0s on the left if necessary. 779 No 01100001011 If you encode an odd number of characters, as we are here, take a numeric representation of the final character and convert it to a 6-bit binary string. Step3: Break up 8-bit Codewords and add Pad Bytes if necessary Step4: Encode these bits with an error correction code.

The following figure shows the generated QR code as a metro train ticket.[20]

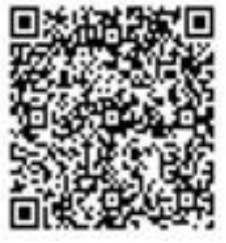

Fig 5. QR code version 7 (as generated)

ii. The pattern generation: Patterns are generated from the passwords given by the users. The passwords as texts are first converted in to bits of length 16 . For the converted bits patterns are generated using the pixilation tool. The generated patterns are then embedded in the QR code that will match the correlation values [1]. The patterns are binary and have $16 \mathrm{x}$ 16 pixel size.

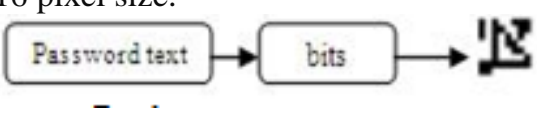

iii.: The colored components in the Unique code are substituted by contoured structures. There is no transition in the Colored destination label packages. The community level of the bi-level Url can be perused by the regular barcode. The private level needs a unique scanner which is application specific.

Information retrieval: The public level of the bi-level $\mathrm{QR}$ code has been scanned by various scanners available in the Google play store. The following figure shows the scanned bi-level QR code which is scanned by the application "QR Scanner".

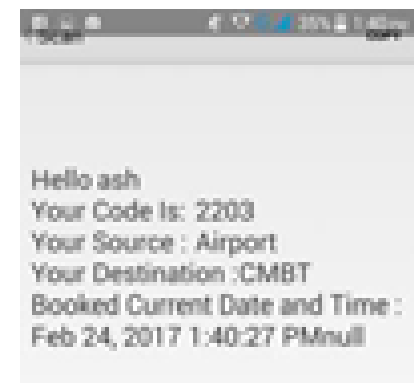

Fig 7. public level information retrieved using $Q R$ code scanner..

We suggest that all errors due to incorrect model recognition are fixed using the Golay missteps adjustment method: one hundred percent of the message numbers are encoded. [1].The private message is retrieved using an application specific scanner which will identify the patterns and decode the pattern. The private message as the payment details is scanned using the unique scanner. The following figure illustrates the retrieval of the public information.

The proposed bi-level QR code evaluation is compared With many current rich visual barcodes in the table below. Only (together with the suggested Bi Level Barcode) the indicated visual designs are tactile to the printing technique, in relation to being the easiest to have personal trunk level features. The first and most studio possibility of Unique code 
with concealed message[2] is the easiest of 9720 bits utilizing Barcode V40.

The sensitivity to copying has been improved in this paper by dynamically generating the patterns which can be changed by the user as and when they require. The early works include including the already designed textured patterns from the pattern database into the black modules of the QR code. The experiment results have shown that the authentication property has been enhanced by dynamically generating the patterns and impeding into the $\mathrm{QR}$ code. Also, when the $\mathrm{QR}$ code is shared via any medium like Bluetooth or any sharing means this authentication will help to store the private information only with the shared user if the QR code has been shared with any unauthorized user.

The suggested bi-level QR code can be used for any QR The tamper evident password. In reality, the original bi-degree is directly attributable to the particular characteristics of the textured genres used. Txt file may be differentiated from one in each of its versions in order to allow certain identification. This capacity implemented due to the impact of the P\&S scheme. where the styles can't be duplicated due to each the Deficiencies in the physical system and the stochastic nature of the matter... Type in anything that you want. Then click Quill It on the right to rewrite your input.

\section{CONCLUSION}

Thus, we say that there are third parties in bi-stage QR program, the first flat can be studied through the aid of any QR program that studies software. second stage, on the other hand, wants a special utility. The $2 \mathrm{~d}$ level is developed with the aid of the use of specific binary contoured styles, which can be distinguished from each other by $\mathrm{P} \& \mathrm{~S}$ and the overall Barcode shall be considered as black parts. In the future, we are planning to follow the capabilities of $\mathrm{Bi}$ - Level QR code, focusing on each QR system and specimen duration, as well as proposing distinct pattern identification algorithms, with a perspective to being less delicate to the $P \& S$ impact.

\section{REFERENCES}

1. Kongkham, D. \& Sundararajan, M. 2019, "Distributed wideband sensing method for faded dynamic spectrum access", International Journal of Innovative Technology and Exploring Engineering, vol. 8, no. 10, pp. 4309-4312.

2. Balaji, S., John Paul Praveen, A. \& Mohanraj, R. 2019, "Recognizable proof and analysis of palm print in biometric authentication system using bayes techniques", International Journal of Innovative Technology and Exploring Engineering, vol. 8, no. 9 Special Issue 3, pp. 1126-1129.

3. Kavitha, G., Priya, N., Velvizhi, R. \& Allin Geo, A.V. 2019, "Parallel computation in correspondence and signal processing", International Journal of Innovative Technology and Exploring Engineering, vol. 8, no. 9 Special Issue 3, pp. 1136-1139.

4. Hema, R., Sundararajan, M. \& Balaji, S. 2019, "Smartphone control robot with automatic firing gun", International Journal of Innovative Technology and Exploring Engineering, vol. 8, no. 9 Special Issue 3, pp. 625-627.

5. Kaliyamurthie, K.P., Sundar Raj, B., Velvizhi, R. \& Shanmugapriya, K. 2019, "Dual band paper substrate CPW antenna for wireless applications", International Journal of Innovative Technology and Exploring Engineering, vol. 8, no. 9 Special Issue 3, pp. 605-608.

6. Geo, A.V.A., Arunachalam, A.R., Michael, G. \& Elankavi, R. 2019 , "Evaluating architecture using compact modalities", International Journal of Innovative Technology and Exploring Engineering, vol. 8, no. 9 Special Issue 3, pp. 836-838.

7. Theivasigamani, S., Jeyapriya, D. \& Anita Davamani, K. 2019, "Anamoly analyzing and exploring for wireless sensor networks",
International Journal of Innovative Technology and Exploring Engineering, vol. 8, no. 9 Special Issue 3, pp. 1116-1118.

8. Jeyapriya, D., Theivasigamani, S., Velvizhi, R. \& Nandhini, P. 2019 "Program detection in wireless feeler networks", International Journal of Innovative Technology and Exploring Engineering, vol. 8, no. 9 Special Issue 3, pp. 1194-1195.

9. Gowri Sankaran, B., Karthik, B. \& Vijayaragavan, S.P. 2019, "Image compression utilizing wavelet transform", International Journal of Innovative Technology and Exploring Engineering, vol. 8, no. 10, pp. 4305-4308.

10. Gowri Sankaran, B., Karthik, B. \& Vijayaragavan, S.P. 2019, "Weight ward change region plummeting change for square based image huffman coding", International Journal of Innovative Technology and Exploring Engineering, vol. 8, no. 10, pp. 4313-4316

11. Hema, R., Sundararajan, M. \& Balaji, S. 2019, "Smartphone control robot with automatic firing gun", International Journal of Innovative Technology and Exploring Engineering, vol. 8, no. 9 Special Issue 3, pp. 625-627.

12. Rangaswamy, K. \& Rajabhushanam, C. 2019, "Congestion control in wireless network using TCP friendly rate control (TFRC)", International Journal of Recent Technology and Engineering, vol. 8, no. 2 Special issue 3, pp. 1598-1602.

13. Tamil Selvan, S. \& Sundararajan, M. 2019, "Performance Parameters of 3 Value 8t Cntfet Based Sram Cell Design Using H-Spice", International Journal of Recent Technology and Engineering, vol. 8 , no. 2 Special issue 5, pp. 22-27.

14. Vinoth, V.V. \& Kanniga, E. 2019, "Steganographical techniques in hiding text images - system", International Journal of Recent Technology and Engineering, vol. 8, no. 2, pp. 6535-6537.

15. Saravana, S., Balaji, S., Arulselvi, S. \& John Paul Praveen, A. 2019 "Reliable power quality monitoring and protection system", International Journal of Innovative Technology and Exploring Engineering, vol. 8, no. 9 Special Issue 3, pp. 644-645.

16. Sundaramoorthy, A. \& John Wiselin, M.C. 2019, "Single patch antenna with multiple feed", International Journal of Innovative Technology and Exploring Engineering, vol. 8, no. 9, pp. 1743-1747.

17. Velavan, R., Bharanidharan, S. \& Sheeba, B. 2019, "EMF pollution Causes, effects and protection", International Journal of Innovative Technology and Exploring Engineering, vol. 8, no. 9 Special Issue 3 , pp. 1166-1168.

18. Veer, R.A., Arulselvi, S. \& Karthik, B. 2019, "Construction of ensemble square classification approaches in MIMO OFDM", International Journal of Engineering and Advanced Technology, vol. 8, no. 5 , pp. 2039-2041.

19. Agitha, W. \& Kaliyamurthie, K.P. 2019, "Improved energy efficient in WBAN using MAC with cloud computing", International Journal of Innovative Technology and Exploring Engineering, vol. 8, no. 8, pp. 2405-2408.

20. Kastro, G.G. \& Wiselin, M.C.J. 2019, "Design and analysis of stub loaded resonator", International Journal of Recent Technology and Engineering, vol. 8, no. 1 Special Issue4, pp. 272-283.

\section{AUTHORS PROFILE}

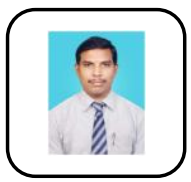

Karthik.B,Assistant Professor, Department of Electronics And Communication Engineering,, Bharath Institute of Higher Education and Research, Chennai, India.

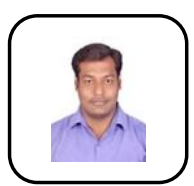

Sriram.M, Assistant Professor, Department of Electronics And Communication Engineering,, Bharath Institute of Higher Education and Research, Chennai.

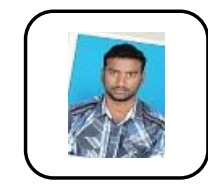

Sidharth Raj.R.S., Assistant Professor, Department of Electronics And Communication Engineering,, Bharath Institute of Higher Education and Research, Chennai, India

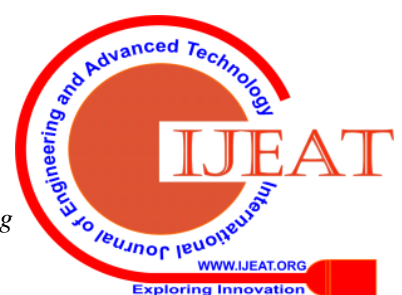

